\title{
Comportamento de Dípteros Muscóides Frente a Substratos de Oviposição, em Laboratório, no Rio de Janeiro, RJ, Brasil
}

\author{
José Mario d'Almeida, Rubens Pinto de Mello *
}

Laboratório de Biologia e Controle de Insetos Vetores, Departamento de Biologia *Departamento de Entomologia, Instituto Oswaldo Cruz, Av. Brasil 4365, 21045-900 Rio de Janeiro, RJ, Brasil

Behavior of Caliptrate Diptera in Relation to the Choose of Oviposition Substrates under Laboratory Conditions in Rio de Janeiro, RJ, Brazil - How adult females of calyptrate Diptera recognize the appropriate breeding substrate is a matter of controversy. Among holometabolic insects, the feeding opportunities of immature stages are generally determined by the adult female choice of an oviposition site.

The ovipositional and larvipositional substrate preference for the synanthropic flies (Chrysomya megacephala, C. putoria, Phaenicia cuprina: Calliphoridae; Atherigona orientalis, Synthesiomyia nudiseta: Muscidae; Ravinia belforti, Parasarcophaga ruficornis, Peckia chrysostoma: Sarcophagidae) is presented in this work. The substrate used for testing were the following: bovine minced meat, fish (sardine), bovine liver, shrimp, squid, human faeces and banana. Bovine minced meat was the ovipositional and larvipositional substrate preferred by seven species. Human faeces were preferred by R. belforti.

Key words: Diptera - ovipositional substrates - synanthropic flies

Os mecanismos que levam um muscóide a ovipor ou larvipor em determinados substratos ainda causam controvérsias. Alguns pesquisadores sugerem que os odores exalados estimulam as fêmeas a ovipor, enquanto outros afirmam que o comportamento de oviposição se relaciona diretamente com a capacidade das moscas de reconhecer os alimentos larvares (Zucolloto 1991). A teoria da "dupla discriminação" sustenta que tanto o valor nutricional dos meios para as larvas, como o odor exalado, agem, conjuntamente, na escolha do substrato de ovipostura pelas fêmeas (Kennedy $\&$ Both 1951). Bay e Pitts (1976) assinalam que em Musca autumnalis para ovipor é necessário, além do odor captado pelas antenas, o contato dos palpos com o substrato, entretanto, Barton-Browne (1960), estudando o comportamento de Phormia regina, observou que para a oviposição basta o estímulo olfatório.

São raros os estudos sobre a preferência de muscóides por substratos de oviposição (Laake et al. 1931, Thomson \& Hammer 1936, Clift \& Mac Donald 1976), apesar da ampla literatura existente sobre a morfologia e a fisiologia dos órgãos olfativos e táteis das moscas (Cragg 1956a, b, Barton-Browne 1960, Bay \& Pitts 1976, Quattro \& Wasti 1978 ).

Parte da Tese de Doutorado do primeiro autor Recebido em 21 de março de 1995

Aceito em 7 de Junho de 1995
Considerando-se a relevância que esses insetos assumem em Saúde Pública, como potenciais vetores de agentes etiológicos de doenças (Greenberg 1971), com o objetivo de estudar o comportamento de alguns dípteros muscóides sinantrópicos, na escolha dos substratos de oviposição, sob condições de laboratório, apresentamos este trabalho em continuidade a outros sobre criadouros de moscas, iniciada em ambientes naturais do Rio de Janeiro (d'Almeida 1986, 1988, 1989, 1993).

\section{MATERIAIS E MÉTODOS}

Colônias de Chrysomya megacephala (Towsend, 1935), Chrysomya putoria (Wiedemann, 1819), Phaenicia cuprina (Wiedemann, 1830) (Calliphoridae); Atherigona orientalis (Schiner, 1860), Synthesiomyia nudiseta (Wulp, 1830) (Muscidade); Parasarcophaga ruficornis (Fabricius, 1794), Peckia chrysostoma (Wiedemann, 1830) e Ravinia belforti (Prado \& Fonseca, 1932) (Sarcophagidae) foram estabelecidas a partir de exemplares capturados com redes entomológicas no Jardim Zoológico do Rio de Janeiro, atraídas por peixe em decomposição.

As posturas das moscas recém-capturadas foram semeadas em carne bovina, para iniciar as colônias. Após as emergências, os insetos de primeira geração foram acondicionados em gaiolas de madeira, revestidas de tela de "nylon", de $30 \mathrm{~cm}$ $\mathrm{X} 30 \mathrm{~cm}$, e alimentados até o quarto dia de vida, com fígado cru e uma mistura de leite em pó, açú- 
car refinado e levedura de cerveja (2:1:1), trocados diariamente, além de água ad libitum. Do quinto dia de vida até o início das experiências no décimo dia, era fornecida apenas a mistura de leite para evitar posturas antecipadas.

Para os experimentos, efetuados sob condições de laboratório $\left(22-28^{\circ} \mathrm{C}, 80 \pm 10 \% \mathrm{UR}\right)$, usaramse dois machos para cada fêmea.

Os ensaios foram efetuados com seis substratos: peixe (sardinha), fígado (bovino), carne (bovina moída), camarão (sem casca), lula, banana fermentada, retirados do freezer e deixados em exposição ao meio ambiente por 32 horas e fezes humanas frescas. Esses substratos, eram moídos e acondicionados em potes de polietileno $(3,5 \mathrm{~cm}$ de altura por $5,0 \mathrm{~cm}$ de diâmetro), aproximadamente 10 gramas por recipiente. Nas gaiolas de experimentação eram apresentados concentricamente e simultâneamente para as fêmeas efetuarem as escolhas. Para cada espécie foram realizadas seis repetições, conforme o desenho experimental da Tabela I; em cada uma delas, os substratos foram expostos, simultâneamente durante quatro dias, com a primeira exposição no $10^{\circ}$ dia após a emergência, e as demais em dias alternados. Obtidas as posturas, as larvas e os ovos eram contados, sendo que os ovos somente após a dissociação do cemento, pela imersão em hipoclorito de sódio a $2 \%$.

As experiências foram desenvolvidas de maio a setembro de 1992, no Laboratório de Biologia e Controle de Insetos Vetores do Departamento de Biologia do Instituto Oswaldo Cruz.

Os resultados obtidos foram analisados estatísticamente, usando-se o teste de aderência do $\chi^{2}$ ( qui - quadrado ), ao nível de $1 \%$ de probabilidade.

\section{RESULTADOS E DISCUSSÃO}

Para efetuar esta pesquisa, utilizaram-se algumas das espécies que obtiveram altos índices de sinantropia, em trabalhos efetuados no Brasil, portanto, insetos de importância sanitária (Ferreira 1978, Linhares 1979, d' Almeida 1983, Dias et al. 1984).

Na Tabela II observa-se o número total de ovos e larvas depositados nos substratos, assim como a média de ovos/fêmea/substrato. A análise estatística da preferência das espécies pelos diferentes meios, de acordo com o número de ovos e larvas, é apresentada na Tabela III. A Tabela IV permite visualizar a frequência com que os meios eram utilizados pelas moscas para larvipor e ovipor.

De um modo geral, a carne foi o substrato onde as fêmeas depositaram o maior número de ovos $\mathrm{e}$ larvas, como também, foi o meio mais freqüentemente procurado para as posturas $(60,9 \%$ das exposicões) (Tabela IV). Fezes e banana não exerceram estímulo para a oviposição nos califorídeos, entretanto, foram procuradas pelos muscídeos e sarcofagídeos. Coffey (1966) pesquisando moscas criadas em diversos substratos, dentre os quais banana, fezes humanas e de variados animais, em Washington, EUA, observou que as fezes de carnívoros estritos são as que funcionam melhor como criadouros de califorídeos. Lopes (1973) assinala que as fezes humanas exercem forte atração sobre os Sarcophagidae, não só para a alimentação, mas também para a criação, e, segundo ainda esse au-

TABELA I

Desenho experimental dos procedimentos adotados para avaliar a preferência de dípteros muscóides pelos subtratos de postura, em condições de laboratório no Rio de Janeiro, RJ

\begin{tabular}{|c|c|c|c|c|c|c|c|c|}
\hline \multirow[t]{2}{*}{ Espécies } & & \multicolumn{6}{|c|}{ Repetições } & \multirow[t]{2}{*}{ Total } \\
\hline & & 1 & 2 & 3 & 4 & 5 & 6 & \\
\hline \multirow[t]{2}{*}{ Chysomya megacephala } & Fêmeas & 17 & 17 & 20 & 13 & 13 & 12 & 92 \\
\hline & Machos & 34 & 34 & 40 & 26 & 26 & 24 & 184 \\
\hline \multirow[t]{2}{*}{ Chysomya putoria } & Fêmeas & 14 & 12 & 13 & 13 & 13 & 13 & 78 \\
\hline & Machos & 28 & 24 & 26 & 26 & 26 & 26 & 156 \\
\hline \multirow[t]{2}{*}{ Phaenicia cuprina } & Fêmeas & 15 & 14 & 15 & 15 & 11 & 13 & 83 \\
\hline & Machos & 30 & 28 & 30 & 30 & 22 & 26 & 166 \\
\hline \multirow[t]{2}{*}{ Ravina belforti } & Fêmeas & 16 & 15 & 16 & 12 & 11 & 12 & 82 \\
\hline & Machos & 32 & 30 & 32 & 24 & 22 & 24 & 164 \\
\hline \multirow[t]{2}{*}{ Parasarcophaga ruficornis } & Fêmeas & 15 & - & - & 13 & 13 & 13 & 54 \\
\hline & Machos & 30 & - & - & 26 & 26 & 26 & 108 \\
\hline \multirow[t]{2}{*}{ Peckia chrysostoma } & Fêmeas & 14 & 12 & 13 & 13 & 13 & 13 & 61 \\
\hline & Machos & 28 & 24 & 26 & 26 & 26 & 26 & 122 \\
\hline \multirow[t]{2}{*}{ Atherigona orientalis } & Fêmeas & 14 & 12 & 13 & 9 & 10 & 9 & 67 \\
\hline & Machos & 28 & 24 & 26 & 18 & 20 & 18 & 134 \\
\hline \multirow[t]{2}{*}{ Synthesiomyia nudiseta } & Fêmeas & 15 & 21 & 18 & 15 & 13 & 13 & 95 \\
\hline & Machos & 30 & 42 & 36 & 30 & 26 & 26 & 190 \\
\hline
\end{tabular}


TABELA II

Números de ovos e larvas de dípteros caliptrados depositados em variados subtratos, sob condições de laboratório, no Rio de Janeiro, RJ

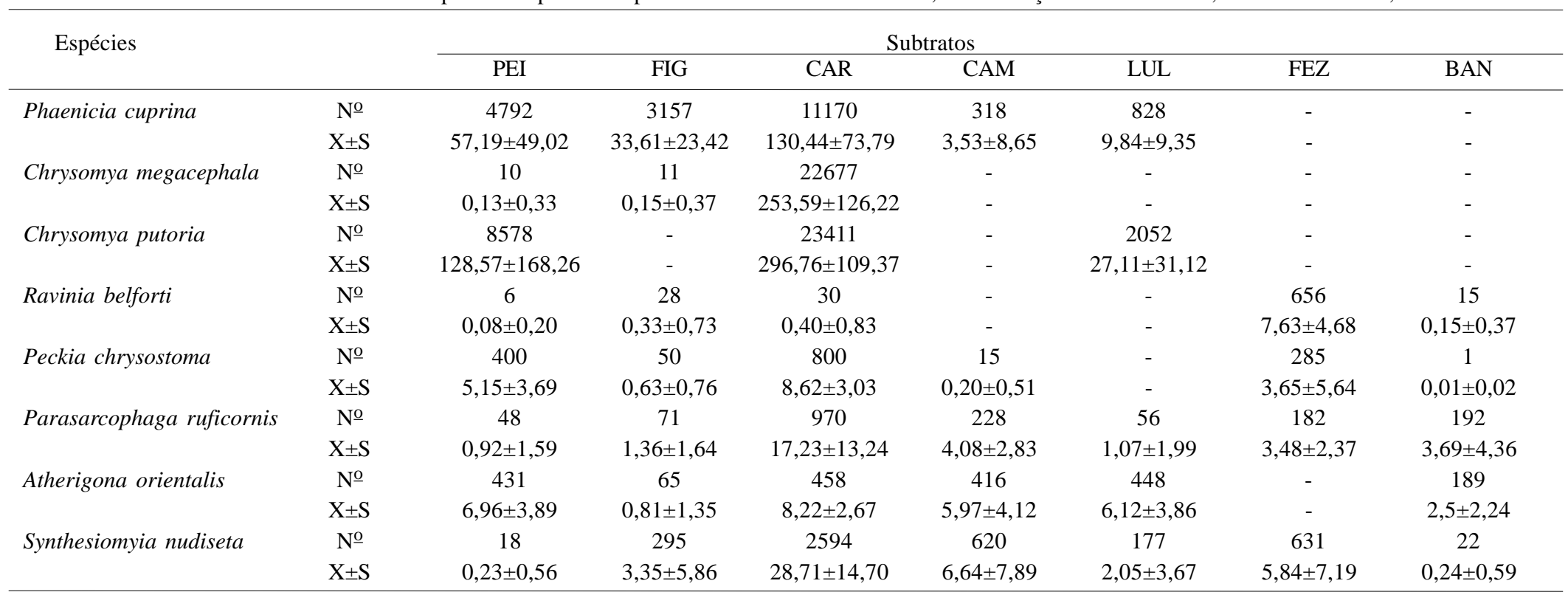

PEI: peixe, FIG: fígado, CAR: carne, CAM: camarão, LUL: lula, FEZ: fezes, BAN: banana; №: número de ovos ou larvas depositados nos substratos; X+S: média e desvio padrão de ovos ou larvas/fêmeas/substrato 
tor (Lopes 1973), algumas espécies dos gêneros Oxysarcodexia e Oxyvinia se criam apenas nas fezes.

Com exceção da A. orientalis, o achado de ovos e larvas em banana, sugere que os muscóides foram apenas estimulados a ovipor, pelos odores exalados pelo substrato, já que esses insetos necessitam de proteína animal para o desenvolvimento larvar (d' Almeida 1988, 1989).

A carne foi o substrato preferido para a oviposição de $P$. cuprina (Tabelas II e III) pois $55,11 \%$ dos ovos foram depositados nesse meio. Foram também efetuadas posturas em $75 \%$ da totalidade de exposições deste substrato, indicando que em carne as oviposições são mais constantes (Tabela IV). Para as espécies do gênero Phaenicia (P. cuprina, P. eximia e P. sericata), o fígado tem se mostrado eficiente, não só como substrato de criação, mas também como isca de atração (Quattro \& Wasti 1978, d' Almeida \& Lopes 1983, d' Almeida 1989, Delves \& Browne 1989). Entretanto, no presente trabalho, apenas $15 \%$ dos ovos de $P$. cuprina foram depositados em fígado, daí concluir-se que este substrato, quando apresentado simultaneamente com outros mais atrativos, estimula pouco a oviposição.

Nesta pesquisa observou-se que $C$. megacephala depositou praticamente todos os ovos em carne $(99,9 \%$ do total). Fezes e banana não estimularam a oviposição; contudo, alguns autores relatam que as fezes são eficientes criadouros de C. megacephala (Thomas 1951, Norris 1965). Os resultados obtidos no presente trabalho, somados aos de outros levantamentos, indicam que este califorídeo não é atraído pelas fezes e, muito menos estimulado a ovipor (Coffey 1966, d' Almeida 1988, 1989).

\section{TABELA III}

Preferência dos dípteros muscóides por substratos de postura. A ordem decrescente é da eaquerda para direita e aqueles que não difererm significativamente, ao nível de $1 \%$ de probabilidade, estão assinanalados por uma

linha horizontal

\begin{tabular}{|c|c|}
\hline Espécies & Substratos \\
\hline Phaenicia cuprina & CAR-PEI-FIG-LUL-CAM \\
\hline Chrysomya megacephala & CAR-FIG-PEI \\
\hline Chrysomya putoria & CAR-PEI-LUL \\
\hline Ravinia belforti & 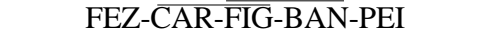 \\
\hline Parasarcophaga ruficornis & CAR-CAM- $\overline{\mathrm{BAN}}-\mathrm{FEZ}-\overline{\mathrm{FIG}}-\overline{\mathrm{LUL}}-\mathrm{PEI}$ \\
\hline Peckya chrysostoma & CAR-PEI-FEZ-FIG-CAM-BAN \\
\hline Atherigona orientalis & 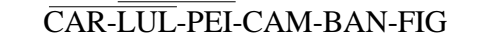 \\
\hline Synthesiomyia nudiseta & 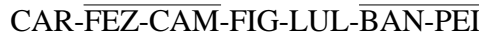 \\
\hline
\end{tabular}

PEI: peixe, FIG: fígado, CAR: carne, CAM: camarão, LUL: lula, FEZ: fezes, BAN: banana

\section{TABELA IV}

Freqüência relativa das oviposições e larvipposições efetuadas nos subtratos expostos nas gaiolas de criação dos dípteros caliptrados, no Rio de Janeiro, RJ

\begin{tabular}{lrrrrrrr}
\hline Espécies & \multicolumn{7}{c}{ Subtratos } \\
\cline { 2 - 8 } & PEI\% & FIG\% & CAR\% & CAM\% & LUL\% & FEZ\% & BAN\% \\
\hline Phaencia cuprina & 50,0 & 45,8 & 75,0 & 4,2 & 20,8 & - & - \\
Chrysomya megacephala & 33,3 & 4,2 & 70,8 & - & - & - & - \\
Chrysomya putoria & 37,5 & - & 62,5 & - & 20,8 & - & - \\
Ravinia belforti & 4,2 & 16,7 & 4,2 & - & - & 95,8 & 8,3 \\
Peckia chrysostoma & 37,5 & 33,3 & 62,5 & 4,2 & - & 25,0 & 4,2 \\
Parasarcophaga ruficornis & 6,2 & 18,7 & 37,5 & 25,0 & 12,5 & 25,0 & 12,5 \\
Atherigona orientalis & 58,3 & 12,5 & 95,8 & 66,7 & 79,2 & - & 45,8 \\
Synthesiomyia nudiseta & 4,2 & 12,5 & 67,7 & 20,8 & 8,3 & 16,7 & 4,2 \\
\hline Total das espécies ${ }^{a}$ & 26,6 & 15,8 & 60,9 & 15,2 & 17,4 & 20,1 & 9,2 \\
\hline
\end{tabular}

PEI: peixe, FIG: fígado, CAR: carne, CAM: camarão, LUL: lula, FEZ: fezes, BAN: banana; $a$ : representativa da frequiência que o substrato foi procurado pelo total das espécies 
C. putoria depositou seus ovos em três dos sete substratos (Tabela II), dentre os quais, a carne foi o preferido (Tabela III), embora não com tanta intensidade como o fez C. megacephala . Segundo Greenberg (1971), C. putoria se alimenta e ovipõe em diversos tipos de materiais orgânicos em decomposição, principalmente carnes e raramente fezes; esta afirmação corrobora os achados da presente pesquisa.

$R$. belforti demonstrou nítida preferência pelas fezes como substrato de larviposição (Tabelas II e III), depositando $90 \%$ do total das larvas neste meio. Convém ressaltar que este sarcofagídeo larvipôs em $95,5 \%$ dos potes de fezes apresentados (Tabela IV). Estes resultados, associados aos de outras pesquisas, onde fezes humanas e de animais foram os melhores criadouros de $R$. belforti (Lopes 1973, d' Almeida 1988, 1989), ressaltam a íntima relação deste inseto com fezes, enaltecendo a sua importância como potencial vetor de enteropatógenos.

Dentre os muscóides avaliados, apenas $S$. nudiseta e P. ruficornis depositaram ovos e larvas em todos os meios expostos, entretanto, a carne foi o substrato preferido (Tabelas II e III). Segundo d' Almeida (1988 e 1989), o fígado foi o substrato de criação mais eficiente para S. nudiseta na área urbana e no Jardim Zoológico do Rio de Janeiro. Comparando-se os achados sobre oviposição com os de criação de $S$. nudiseta, chega-se a uma conclusão semelhante àquela obtida para $P$. cuprina.

Em $P$. chrysostoma além da carne, o peixe também exerceu forte estímulo para a larviposição (Tabelas II e III), o que está de acordo com os resultados obtidos por d' Almeida (1986 e 1988) nas áreas rural e urbana, respectivamente. Lopes (1973) também criou esta espécie em peixe na Floresta da Tijuca, no Rio de Janeiro.

Convém ressaltar que apenas em A. orientalis não ocorreram diferenças significativas, ao nível de $1 \%$ de probabilidade, entre o número de ovos depositados na carne e na lula, outro substrato muito procurado por este muscídeo; isto sugere que os dois meios estimulam igualmente a oviposição (Tabela III). Segundo Morón e Terrón (1984), a lula é uma boa isca de atração por apresentar decomposição rápida e fetidez perdurável. Esta espécie foi a que utilizou com maior regularidade os substratos expostos, em especial a carne, onde efetuou posturas em $95,5 \%$ dos recipientes. d' Almeida (1989) afirma que A. orientalis se desenvolve intensamente na matéria orgânica vegetal em decomposição, entretanto, em levantamentos faunísticos é também atraída com freqüência por matéria orgânica animal em decom- posição (Linhares 1979, d' Almeida 1983).

Comparando-se os resultados das Tabelas II e IV pode-se notar que a maioria das espécies, apesar de demonstrar nítida preferência por determinados substratos, não fez regularmente posturas nestes meios. Entretanto $R$. belforti e A. orientalis distribuiram com mais regularidade as larvas e ovos nos substratos. Essas constatações possivelmente podem estar associadas à fatores como potencial reprodutivo e estratégia de sobrevivência, dentre outros, como já foi verificado com $R$. belforti (d'Almeida, entregue para publicação), que demostrou baixo potencial reprodutivo como estratégia de sobrevivência.

De acordo com os resultados obtidos, as seguintes conclusões podem ser apresentadas: a carne foi o substrato preferido por sete das oito espécies de dípteros muscóides estudados; fezes humanas foi o substrato preferido pela $R$. belforti, o que exalta a possível importância sanitária desta espécie; C. megacephala praticamente só ovipôs em carne $(99,90 \%)$; S. nudiseta e P. ruficornis fizeram oviposições e larviposições, respectivamente, em todos os substratos apresentados.

\section{REFERÊNCIAS}

Barton- Browne L 1960. The role of olfaction in the stimulation of oviposition in the blowfly, Phormia regina. J Insect Physiol 5: 16-22.

Bay DE, Pitts CW 1976. Olfactory responses of face flies Musca autumnalis to bovine faeces. Ann Entomol Soc Am 69: 933-936.

Clift AD, Mac Donald FJD 1976. Some relationships between diet and ovarian development in Lucilia cuprina (Diptera: Calliphoridae). Aust J Zool 24: 87-93.

Coffey MD 1966. Studies on the association of flies (Diptera) with dung in Southeastern Washington. Ann Entomol Soc Am 59: 207-218.

Cragg JB 1956a. The olfactory behaviour of Lucilia species (Diptera) under natural conditions. Ann Appl Biol 44: 467-477.

Cragg JB 1956b. The action of climate on the larva, prepupae and pupae of certain blowflies. p. 387389. Proc Intern Congr Zool 14th. Copenhagen, 1953.

d'Almeida JM 1983. Sinantropia em dípteros caliptrados na área metropolitana do Rio de Janeiro. Tese de Mestrado, UFRRJ, Rio de Janeiro, 193 pp.

d'Almeida JM 1986. Substratos utilizados para a criação de dípteros caliptrados em uma área rural do Estado do Rio de Janeiro. Arq Univ Fed Rur Rio de J 9: 13-22.

d'Almeida JM 1988. Substratos utilizados para a criação de dípteros caliptrados em uma área urbana do Município do Rio de Janeiro. Mem Inst Oswaldo Cruz 83: 201-206.

d'Almeida JM 1989. Substratos utilizados para a criação de dípteros caliptrados no Jardim Zoológico do Rio de Janeiro (RIO- ZOO). Mem Inst Oswaldo 
Cruz 84: 257-264.

d'Almeida JM 1993. Capture of calliptrate flies with different breeding substrates on beaches in Rio de Janeiro, RJ, Brazil. Mem Inst Oswaldo Cruz 88: 215-220.

d'Almeida JM, Lopes HS 1983. Sinantropia em dípteros caliptrados (Calliphoridae) no Estado do Rio de Janeiro. Arq Univ Fed Rur Rio de J 6: 3848.

Delves RI, Browne LB 1989. Changes in reproduction performace of the australian sheep blowfly, Lucilia cuprina (Diptera, Calliphoridae) following laboratory colonization. J appl Entomol 107:228237.

Dias ES, Neves DP, Lopes HS 1984. Estudos sobre a fauna de Sarcophagidae (Diptera) de Belo Horizonte, MG: I- levantamento taxonômico e sinantrópico. Mem Inst Oswaldo Cruz 79: 83-91.

Ferreira MJM 1978. Sinantropia de dípteros muscóides de curitiba, Paraná. I. Calliphoridae. Rev Brasil Biol 38: 445-454.

Greenberg B 1971. Flies and Diseases vol. I: Ecology, classification and biotic associations: Princeton Univ. Press. Princeton, N. J. XII + 856 pp.

Kennedy JS, Both CO 1951. Feeding preference and fecundity in relation to the age and hing of leaves. Ann Appl Biol 38: 25-64.

Laake EN, Parman DC, Bishopp FC, Roach RC 1931. the chemotropic responses of the house fly, the green bottle fly and the black blowfly. USDA. Tec Bull 270: $1-10$

Linhares AX 1979. Sinantropia de dípteros muscóides de Campinas, Tese de Mestrado, UNICAMP, Campinas, SP. 129 pp.

Lopes HS 1973. Collecting and rearing Sarcophagidae flies (Diptera) in Brazil during forty years. An Acad Bras Ci 45: 279-291.

Morón MA, Terrón R 1984. Distribuición altitudinal de los insectos necrófilos en la sierra norte de hidalgo“ méxico. Acta Zool Mex (n. s.) 3: 1-47.

Norris KR 1965. The bionomics of blowflies. Ann Rev Entomol 10: 47-68.

Quattro MJ , Wasti SS 1978. Olfactory and oviposition responses of the green botlle fly, Phaenicia sericata to a variety of natural baits (Diptera: Calliphoridae). Rev Brasil Biol 8: 115-119.

Thomas HT 1951. Some species of blowflies genera Chrysomya R-D; Lucilia R-D; Hemipyrellia Thed and Calliphora R-D. from south eastern Szechwan, China. Proc Zool London 121: 147-200.

Thomson M, Hammer O 1936. The breeding medium of some common flies. Bull. Entomol Res 27: 559587.

Zucoloto FS 1991. Effects of flavour and nutritional value on diets selection by Ceratites capitata larvae (Diptera: Tephritidae). J Insect Physiol 37: 2125 . 